\title{
Minimizing Building Electricity Costs in a Dynamic Power Market: Algorithms and Impact on Energy Conservation
}

\author{
Dawei Pan ${ }^{\dagger *}$, Dan Wang ${ }^{* \ddagger}$, Jiannong Cao*, Yu Peng ${ }^{\dagger}$, Xiyuan Peng ${ }^{\dagger}$ \\ ${ }^{*}$ The Hong Kong Polytechnic University \\ $\{$ csdwang,csjcao\}@compcomp.polyu.edu.hk \\ ${ }^{\dagger}$ Harbin Institute of Technology \\ \{pandawei,pengyu,pxy\}@hit.edu.cn \\ ${ }^{\ddagger}$ The Hong Kong Polytechnic University Shenzhen Research Institute
}

\begin{abstract}
Energy is a global concern and the electricity bills nowadays are leading to unprecedented costs. Electricity price is market-based and dynamic. In this paper, we investigate how to cut the electricity bills of commercial buildings in a dynamic power market. The building thermal systems (e.g., air-conditioning), which dominate electricity bills, has a special property of thermal storage, i.e., the energy will not immediately dissipate from thermal air/water. Intuitively, with storage, the energy can be "stored" in the thermal system, making it possible to purchase electricity in low price and use it at appropriate time. The building thermal supply and electricity purchasing surely depends on human activities that the building should support such as class and meeting schedules. To minimize electricity bills, we develop a holistic planning of electricity purchasing schedule with thermal storage management, and appropriate room assignment schedules for classes/meetings usage.

The computing algorithms require inputs of physical modeling on energy consumption. We develop wireless sensing systems to collect fine-grained data which are used to assist the cross-disciplinary physical modeling. We conduct validation through real experiments. We formulate an optimization problem and show that it is NP-complete. Our primary focus is to minimize electricity bills, which matches the incentives of the commercial buildings. We show that this does not coincide with energy conservation. We further investigate the relationship of minimization of electricity bills and minimization of energy consumption. We develop algorithms for our problem and our evaluation shows that we can achieve a $40 \%$ cost reduction.
\end{abstract}

\section{INTRODUCTION}

Energy is a global concern nowadays and the energy price is expected to continuously increase. Electricity prices also fluctuate. This is because some power plants cannot stop power generation or some power sources are dynamic (e.g., solar), and peak hour demand leads not only to more electricity loss in power generation/delivery but also to power plant damage and fast deterioration [1]. As such, dynamic price can encourage usage in low demand time and penalize usage in high demand time. The recent development of smart grids aims at diversified electricity generation and fast response to demands [2][3]. A more dynamic power market is widely expected.

An important edge system of the grid is the commercial building. It is one of the four dominating energy consuming sectors, along with transportation, manufactory and resi- dential usage [4]. For regions like Hong Kong, where the Industry sector is small, $65 \%$ of electricity is reported to go to the commercial buildings [5]. In buildings, the thermal systems (i.e., the heating, ventilation and air-conditioning systems, HVAC systems) dominate electricity bills. As an example, it is reported that for the Office Segment of Hong Kong, 54\% electricity goes to space conditioning (i.e., airconditioning), $14 \%$ goes to lighting, $13 \%$ goes to office equipments [5]. In this paper, we investigate how to cut the electricity bills of commercial buildings in a dynamic power market.

The thermal system has a special property of thermal storage. For example, chilled water system is very typical in buildings and the energy will not immediately dissipate from thermal water. Nowadays, even separated Thermal Energy Storage (TES) systems exist [6]. Intuitively, with storage, the energy can be "stored" in the thermal system for a certain time, making it possible to purchase electricity in low price and use it at appropriate time. The building thermal supply and electricity purchasing from the power market surely depends on human activities that the building should support where the human activities could be represented by class or meeting usage schedules. To minimize electricity bills, we need a holistic planning of electricity purchasing schedule with thermal storage management, and appropriate room assignment schedules for classes/meetings usage.

Clearly, this planning falls into an optimization problem. We need carefully designed algorithms. In addition, the computing algorithms require inputs of the thermal consumption of rooms and thermal storage capacity of the HVAC system in the buildings. These require cross-disciplinary physical modeling.

In this paper, we develop a wireless sensing system to collect fine-grained data which are used to assist crossdisciplinary thermal modeling. We validate our physical modeling through real experiments. We formulate an optimization problem to minimize the total electricity bills where we need to develop a schedule for electricity purchasing from the power market and a schedule for meetings and room assignment. We show such problem is NP-complete. Our primary focus in this paper is to minimize electricity bills; this matches the incentives of the commercial 
buildings. We observe, however, that such minimization does not coincide with energy conservation. Intuitively, the optimization may schedule a meeting to a room at a time that can result in low cost, yet high energy consumption. We thus further study the root cause and the correlation between energy consumption minimization and electricity bill minimization. We develop a heuristic algorithm for the overall problem using a Lagrangian relaxation-based method. We conducted comprehensive evaluation based on real pricing data and we see up to a $40 \%$ cost saving as compared to typical current scheduling.

The remaining part of the paper is organized as follows. We discuss related work in Section II. We then present background on building thermal systems and an overview of our problem and solutions in Section III. In Section IV, we formally formulate our problem and analyze its complexity. Before we go into the detailed physical thermal modeling and computing algorithm designs, we discuss the relationship between minimizing electricity cost and minimizing energy consumption in Section V. In Section VI, we present the thermal modeling, wireless sensing system development and experimental validation. Our algorithms are shown in Section VII. In Section VIII, we evaluate our algorithms and finally we conclude our paper in Section IX.

\section{RELATED WORK}

With global concerns on energy conservation, energy price is expected to continuously increase, leading to unprecedented electricity bills in many domains. Electricity grids adopt dynamic pricing strategy to reduce energy loss, minimize power plant damage, etc [7]. There are studies that take advantage of dynamic pricing to reduce bills for data centers. Two early schemes were proposed to reduce the electricity costs by shifting workload of data center from locations with high electricity prices to those with low prices [8]. Following these, a set of algorithms and game theoretical models were developed for various scenarios [9][10]. These studies provide useful experiences. However, building thermal systems have unique characteristics and different background context.

An early work that takes advantage of thermal storage and real time pricing to save electricity bill in commercial buildings is [11]. The work considers the buildings as a whole. They do not study detailed building activity management nor they reveal the conflict between the energy minimization and electricity bill minimization. In a recent work [12], battery is proposed to be used as storage for residential houses. Excellent machine learning techniques are developed to predict next-day consumption. The objective of the paper is also minimizing electricity bills. We differ from them as we consider the storage of the thermal systems and our work focus more on a building/campus environment. The thermal system has greater capacity and is also cheaper. In addition, we develop meeting and room assignment schedules. We have a previous study [13] where we observe that the cool air in a room will not dissipate immediately after a class and class schedules should take such advantage. A followup work develops more refined schedules [14]. These studies only consider the thermal storage of a room, which is small and less practical in real world. In addition, we clearly specify the mismatch between minimizing electricity bills and minimizing energy consumption and we hope this may contribute insights for future studies to search for a balance. As buildings are key edge systems for smart grids, the mismatch shows a concrete example that the smart grid pricing strategies may quantitatively take into consideration; the pricing strategies of smart grids are heavily studied recently [15], yet usually from a high level game theoretical point of view.

As the commercial building is one top energy consuming area [16], there are many other studies contributed by the computer society in recent years: 1) there are studies on fine-grained monitoring systems using the recent advances in wireless sensor networks [17]. An auditing network is built to collect electricity readings [18] and sMAP is developed [19] as a general common layer to record physical information for different applications. Similar systems include [20]. We develop our own testbed where we convert the wired building management systems into wireless without changing upper layer building operational protocols [21];2) there are studies on physical modeling of the building thermal systems [22][23][24]; with an aim to better understand cyber-physical co-designs and 3) there are algorithms on wise and automatical device turning-off to save electricity [25][26], assisted by fine-grained data collection and/or thermal modeling, inference on human presence [27], or human participatory sensing/voting for thermal comfort [28].

\section{BACKGROUND AND AN OVERVIEW}

In this paper, we will use air-conditioning in our presentation for the sake of conciseness (our work straightforwardly handles the heating). A typical HVAC system (the thermal system) [29] has a cold water tank. It is chilled to certain temperatures from time to time and this chilling process consumes huge electricity. Hot air impacts on the chilled water system and is compressed in the supply ductwork. If a room turns on air-conditioning, the ventilation of the room (e.g., VAV box) opens and the cold air is squeezed into the room. The cold air gradually gets heated and returns to circulation. Nowadays, advanced and separated Thermal Energy Storage (TES) system are widely used and this can substantially increase the thermal storage capacity. Intrinsically, thermal storage, including the TES system, is based on the ability of water to store large amount of heat or cold. It is common that the thermal storage system today can reasonably provide short term (less than 2 hours) or even long term (greater than 10 hours) cooling energy [6]. 


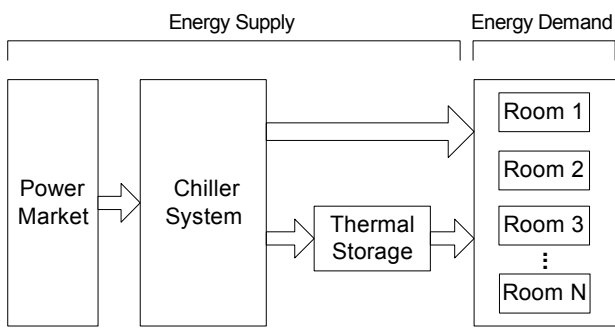

Figure 1: The diagram of the thermal energy flow Physical Computing

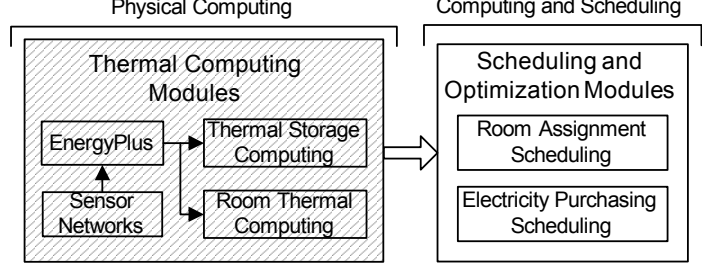

Figure 2: The framework of the thermal cost management system

For a specific room, the amount of electricity it consumes depends on many factors. Two rooms of the same capacity (the number of people the rooms can accommodate) may consume different amount of electricity due to different configurations and/or orientations. At different times of a day, a room also may consume very different amount of electricity.

The energy supply and demand in a building can be abstracted as Fig. 1. The energy demands come from the rooms when scheduled to hold human activities, i.e., meetings. This meeting is meant to be general. In a campus context, this can be translated into class schedules and in a commercial building context, this can be translated into office planning and meeting schedules. We will see in our formulation (Section IV) that a meeting is only associated with the number of people attending the meeting (one person is fine if it is his office room) and a time period (which can be considered as his/their activity patterns). The energy supplies come from the chiller system and the thermal storage. The chiller system is electrically charged to support the energy demands from rooms when the thermal storage is low. All of these finally are electrically supported by the power market. Minimizing building electricity bills in the aforementioned system falls into an optimization problem. Yet we face difficulties both in algorithm design and in physical thermal modeling. Our solution framework is in Fig. 2. On the computing side, we need to develop two schedules: 1) meeting/class schedule (if the time of the meetings is not required to be fixed) and room assignment schedule for meetings; and 2) electricity purchase schedule from the dynamic power market. On the physical side, we need to model: 1) the thermal storage model, and 2) the energy (electricity) requirement for each room if they are assigned for meetings/classes.

The linkage between the computing side and the physical side is that computing schedules need inputs from the physical side. From a high level point of view, we will develop equations that link the dynamics between the demands (room air-conditioning), and the supplies (thermal storage and electricity charges for the chiller system); as we can see from Fig. 1.

We give a more detailed overview of our physical side design in Fig. 2. Thermal computing falls into the expertise of Building and Service Engineering. They have sophisticated tools such as EnergyPlus [30]. One may fill in the parameters of a room (or the thermal systems) and EnergyPlus will output the energy requirements. In EnergyPlus, one can even input the weather of the day, and EnergyPlus can estimate the temperature, solar energy strength according to the weather and output more accurate energy requirements if a room is in use, based on its well trained model and comprehensive history data. EnergyPlus is a complex model and there can have hundreds of parameters.

Our physical computing is based on EnergyPlus. For thermal storage model, we use EnergyPlus directly as it is stand-alone and can be computed once for all. For rooms, we can also use EnergyPlus directly. However, the rooms are very different in configurations. This may introduce high complication if we need to find out the parameters to be input to EnergyPlus room-by-room. Our approach is a wireless sensor system assisted approach as follows. The major complexity comes from some compound parameters that are not easy to obtain directly. As an example, a key parameter is thermal conductivity of a wall. It is difficult to compute from theory as it involves knowledge of subparameters of materials etc, especially, if we have to do it room-by-room for all rooms. We found that these parameters are invariants as it will not change subject to environments. We can inversely calibrate it if we can first collect a set of data on electricity usage, temperature of the room, etc. We develop wireless sensor networks to collect these data. We thus can substantially reduce the number of parameters to be input to EnergyPlus.

\section{The Problem And Its Complexity}

\section{A. The Problem}

Our problem is to compute the meeting and room assignment schedules and the electricity purchasing schedules from the power market, so as to minimize the cost. We now formally formulate this. Assume we have $N$ rooms and $M$ meetings. Let $r_{i}$ and $m_{j}$ denote room $i$ and meeting $j$ respectively.

Without loss of generality, we simplify the meeting requirement to the number of people of the meeting only. We may have additional constraints, such as specific equipments in a room, distance between two meetings/classes in location so that people can travel between the rooms in time, etc. From an optimization point of view, these add more constraints to the problem. Let $w\left(m_{j}\right)$ denote the capacity requirement (number of people) of meeting $m_{j}$. Let $w\left(r_{i}\right)$ denote the capacity of room $r_{i}$. Let $t_{j}^{s}$ and $t_{j}^{e}$ be the meeting 
time is for each meeting $j$. Note that we can have fix meeting times (as requirements/constraints) and/or flexible meeting times (to be computed); and we will study both of them in this paper.

We also simplify the total electricity consumption of a building to be the sum of the electricity consumption of the rooms in supporting meetings. There are surely other electricity consumptions, e.g., lighting, and air-conditioning of the corridors, etc. We argue that the electricity consumption of lighting, etc is much less than air-conditioning; and the air-conditioning of corridors, etc is easy to compute as their usage is regular and stable. Let $\mathcal{E}\left(i, t_{j}^{s}, t_{j}^{e}, \mathcal{T}_{j}\right)$ be electricity consumption of room $i$ at start time $t_{j}^{s}$ and end time $t_{j}^{e}$ with a target temperature $\mathcal{T}_{j}$; e.g., in Hong Kong the recommended temperature for Grade A buildings is $23.5^{\circ} \mathrm{C}\left(74.3^{\circ} \mathrm{F}\right)[31]$.

Let $P_{t}$ be the electricity price at time $t$. Let $V_{t}$ be the thermal storage at time $t$. Let the maximum and minimum thermal storage capacity be $V_{\max }$ and $V_{\min }$.

There are two schedules we need to compute. Let $L_{t}$ be the electricity charge needed at time $t . L_{t}$ represents the electricity purchase schedule. For meeting schedules, we need to decide the room and the meeting time (if the meeting time is pre-determined, this becomes a constraint). Let $x_{i j}$ be an indicator variable, where $x_{i j}=1$ represents that meeting $m_{j}$ is assigned to room $r_{i}$ and 0 otherwise.

Our Minimize Building Electricity Cost (MBEC) problem can be formalized as:

$$
\min \sum_{t} L_{t} P_{t},
$$

1) Meeting Schedule Constraints:

$$
\begin{aligned}
\sum_{i=1}^{N} x_{i j} & =1 \forall j=1, \ldots, M \\
\sum_{j \in \mathcal{J}_{t}} x_{i j} & \leq 1 \quad \forall i=1, \ldots, N \\
w\left(m_{j}\right) & \leq w\left(r_{i}\right) \forall x_{i j}=1
\end{aligned}
$$

2) Thermal Consumption Constraints:

$$
\begin{aligned}
H_{t} & =\sum_{i=1}^{N} \sum_{j \in \mathcal{J}_{t}} x_{i j} \mathcal{E}\left(i, t_{j}^{s}, t_{j}^{e}, \mathcal{T}_{j}\right) \\
H_{t} & \leq H_{\max } \leq L_{\max }
\end{aligned}
$$

3) Thermal Balance Constraints:

$$
\begin{aligned}
V_{t+1} & =a V_{t}+b L_{t}-c H_{t}+d \\
V_{\min } & \leq V_{t} \leq V_{\max }
\end{aligned}
$$

The objective function is self-explanatory. The Meeting Schedule Constraints show that a meeting must be assigned once but the only once, a room can only accommodate one meeting at any time $t$ and the meeting should not exceed the room capacity. Here, $\mathcal{J}_{t}$ represent the set of all meetings that in action at time $t$. Let $H_{t}$ be the total thermal consumption at time $t$. It is computed in the Thermal Consumption Constraints. The thermal consumption at any time must be less than the thermal supply capacity of the HVAC system. This is ensured by design of HVAC system. The Thermal Balance Constraints show a state transition equation (more details in Section VI) should be maintained between each time $t$ and $t+1$. Intuitively, the thermal storage at time $t+1$ equals the thermal storage at time $t$, plus electricity charges and minus electricity usage. $V_{t}$ has an upper and lower limit at any time $t$. The state coefficients $a, b, c, d$ is related to thermal efficiency and loss.

In this problem, the inputs of $\mathcal{E}\left(i, t_{j}^{s}, t_{j}^{e}, \mathcal{T}_{j}\right)$ and the state coefficients need to be computed from the physical side.

\section{B. Complexity analysis}

Theorem 1: MBEC is NP-complete.

Proof: The proof reduces Job Interval Selection Problem [32] to MBEC. The detail of the proof is in [29].

Another problem that is of interest is to have the meeting start and end time fixed. We call this problem f-MBEC. fMBEC is also NP-complete, we reduces Cost Constrained Fixed Job Scheduling [33] to f-MBEC, see our proof in [29]. We specially mention f-MBEC because 1) f-MBEC is practical in many scenarios and 2) f-MBEC is quite different from MBEC in the NP-complete proof and analysis. We comment, in high-level, on the difference between MBEC and f-MBEC. The key complexity difficulty of MBEC and f-MBEC comes from the meeting scheduling (similar to job scheduling). There are two different types of job scheduling: one is finding a schedule to satisfy the timing of all jobs [32] and one is minimization cost for fixed jobs [33]. The complexity reduction are from completely different threads.

In this paper, we mainly focus on MBEC. In Section VII, our algorithm for MBEC surely solves f-MBEC and we will evaluate both in Section VIII.

\section{Electricity COST vs. ENERGy}

Before we go into the details of our algorithms for MBEC and the physical modeling, we first analyze the relationship between minimizing electricity cost and minimizing energy consumption. As said, these two minimizations do not coincide with each other. Note that this is true for both MBEC and f-MBEC, i.e., even the start time and end time of the meetings are fixed, the two minimizations are still not coincide with each other. In this paper, we study the root cause that leads to the differences between the two minimization and in what conditions the two minimizations become identical. It is not the objective of this paper to find a good compromise of them; we believe that this is a grand problem that is also related to power market pricing designs. There are some studies on high-level abstraction [34] and we also plan a future work.

Observation 2: Without real-time pricing, minimizing electricity cost and minimizing energy consumption are identical. 


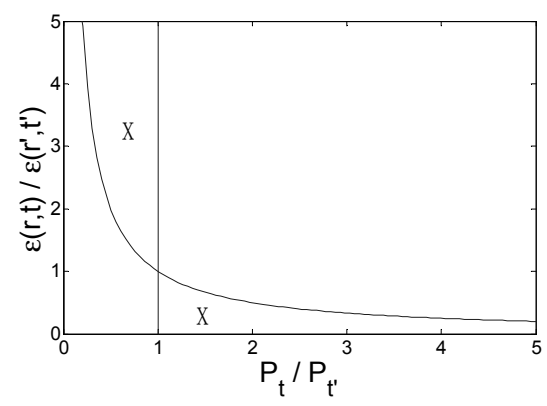

Figure 3: Energy vs. Cost.

Observation 3: If, at any time, all the rooms have identical energy consumption, minimizing electricity cost and minimizing energy consumption are identical.

Intrinsically, if there is no price difference on the supply side, or there is no difference on the demand side, saving cost can be achieved only by saving energy. Thus, the two minimizations become identical. The starting time, ending time of meetings, room capacity, etc are not essential conditions. As a consequence, both MBEC and f-MBEC face that minimizing electricity cost may not conserve energy.

Let $\mathcal{E}(r, t)$ be the energy consumption of room $r$ at time $t$. Define cost-energy in-conflict condition as:

(1) Given $\mathcal{E}(r, t)>\mathcal{E}\left(r^{\prime}, t^{\prime}\right)$ and $P_{t}<P_{t^{\prime}}, \forall r, t, r^{\prime}, t^{\prime}$, $\mathcal{E}(r, t) P_{t}>\mathcal{E}\left(r^{\prime}, t^{\prime}\right) P_{t^{\prime}} ;$ or (2) Given $\mathcal{E}(r, t)<\mathcal{E}\left(r^{\prime}, t^{\prime}\right)$ and $P_{t}>P_{t^{\prime}}, \forall r, t, r^{\prime}, t^{\prime}, \mathcal{E}(r, t) P_{t}<\mathcal{E}\left(r^{\prime}, t^{\prime}\right) P_{t^{\prime}}$.

We use the cost-energy in-conflict condition to capture the essence of Observation 2 and 3. Basically it ensures that energy and electricity cost increase and decrease in the same direction. Therefore,

Lemma 4: If cost-energy in-conflict condition holds, minimizing electricity cost and minimizing energy consumption are identical.

Proof: The detailed proof is in [29].

In Fig. 3, we show an illustration of the cost-energy in-conflict condition. The $\mathrm{X}$-axis is the ratio between the electricity price at any time and the Y-axis is the ratio between the energy consumption of any room at any time. " $\mathrm{X}$ " shows the region where there will be cost-energy conflict. More specifically, if the electricity pricing and/or energy differences of rooms falls into these two regions, minimizing electricity cost and minimizing energy consumption are not the same.

We next show the role that thermal storage can play.

Lemma 5: Given that the thermal storage has infinite capacity, minimizing electricity cost and minimizing energy consumption are identical.

Proof: The detailed proof is in [29].

This lemma shows that thermal storage can mask the difference between the two minimizations. Intuitively, thermal storage hides the impact of the electricity price difference at different times.

To quantitatively understand the difference of the two minimization, we plot an illustration in Fig. 4. The back-

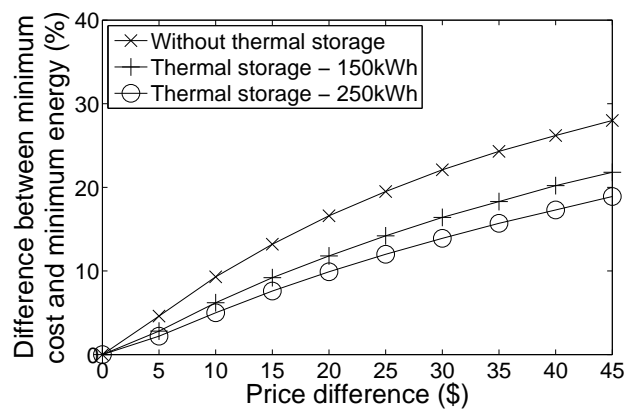

Figure 4: Difference between minimizing electricity and minimizing energy consumption as against to the price difference.

ground is as follows. There are 40 rooms and each room has a capacity to accommodate 60 people. Nevertheless, the energy requirement of each room is different as there are different orientations of the room (more details of how a room can be modeled are in Section VIII-A). We have 250 meetings each with a length of one hour. We compare the solutions for minimizing electricity cost and minimizing energy consumption. We see that when the price difference is $\$ 25$, there can be a difference of around $20 \%$. With a thermal storage capacity of $150 \mathrm{kWh}$, the difference is $15 \%$. Note that the thermal storage capacity of $150 \mathrm{kWh}$ in our setting indicates that the thermal storage can hold for all the building rooms in operation for 1 hour. This is reasonable practice in real world [35] and $\$ 25$ - $\$ 30$ price differences are also quite conservative.

In this paper, we do not further study how we may choose or find a trade-off between electricity bill reduction and energy conservation. We believe there can be separate studies both in a trade-off in building management and in smart grid pricing strategies. In what follows, we emphasize on electricity bill reduction as this matches the incentives of the building operators.

\section{THERMAL COMPUTING}

We now study how we obtain the key physical inputs for our scheduling algorithms. We first present our physical modeling. We present our design and implementation of a wireless sensor network, which is used for data collection to assist our physical modeling. We further present our validation.

\section{A. Physical Modeling}

We first discuss the state transition equation, i.e., Eq. 6 in Section IV. We then discuss how to model thermal storage, i.e., $a$; and energy consumption for rooms, i.e., $\mathcal{E}\left(i, t_{j}^{s}, t_{j}^{e}, \mathcal{T}_{j}\right)$.

1) The State Transition Equation: Let $\dot{L}$ be the electricity charging rate, and $\dot{H}$ be the thermal demand rate. Let $\Delta V$ be the thermal storage charge/discharge volume in a period $\Delta T$. Thus, the thermal storage volume $V$ can be characterized by an electricity charging/thermal demand rate in the following 
expression (the development of such equation follows [29]).

$$
\begin{aligned}
& \dot{L} \Delta T-\dot{H} \Delta T= \pm \Delta V \\
& |\Delta V|<V_{\max }
\end{aligned}
$$

When the electricity charging is greater than the thermal demand, the thermal storage is in the charge mode; and otherwise, in the discharge mode.

In this paper, we transform it into a discrete model by discrete the time:

$$
V_{t+1}=a V_{t}+b L_{t}-c H_{t}+d
$$

We call Eq. 9 the state transition equation. It establishes a linkage between the electricity charging, thermal demand, and the thermal storage; where the electricity charging and thermal demand should be determined by the schedule for electricity purchasing and the schedule for meetings and room assignment respectively.

2) Modeling Thermal Storage and Rooms: We use EnergyPlus for modeling both the thermal storage and room energy requirement since it has extensively tested HVAC modules. Many past experiences on EnergyPlus can be found in [36][14].

As said, we directly use EnergyPlus to model the thermal storage as it is once for all. We can also get the state coefficients $a, b, c, d$ by regression analysis of data of electricity charging and thermal demand from EnergyPlus [11][37]. For energy consumption of the rooms, the number of parameters to be used for EnergyPlus increases fast as rooms are very different. The parameters can be broadly classified as: 1) length, width, and height of the rooms etc. The values of these parameters are easy to obtain, 2) the conductivity of walls etc. These parameters are compound parameters, i.e., further related to materials etc. They are difficult to compute directly but they are invariants, i.e., do not change from environments; and 3) solar radiation, human activity, etc. These changes frequently. Fortunately, however, EnergyPlus has extensive training for these parameters. For example, given the weather, we can get them by linear regression on the historical data from EnergyPlus.

We mainly need to deal with 2). Though EnergyPlus can also be used for 2) it can become over complex due to a large number of rooms. As such, we derive these parameters by inverse calibration. We use thermal conductivity as an example to explain our idea. Let $\lambda$ be the thermal conductivity of the walls. ${ }^{1}$ Ultimately, we want to compute energy requirement of the room $\mathcal{E}$. $\mathcal{E}$ can be written as an equation $\mathcal{E}=f(\lambda, a, b, c, \ldots)$ where $a, b, c, \ldots$ are side parameters that are easy to obtain. To inversely calibrate $\lambda$, we can first collect a set of values of $\mathcal{E}, a, b, c, \ldots$ and inversely solve

\footnotetext{
${ }^{1}$ This can be considered as an average to represent the thermal conductivities of all walls. More specifically, though the walls (including ceiling and floor) of a room $r$ are also different, we can develop a virtual room that has the same energy requirement and property of room $r$; the $\lambda$ of this virtual room well represents the $\lambda \mathrm{s}$ of the walls of room $r$.
}

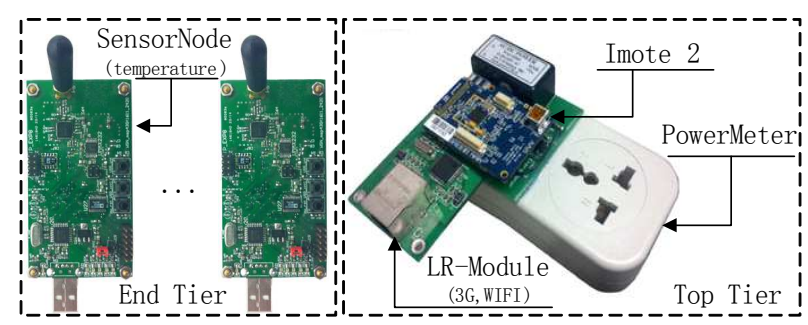

Figure 5: The Sensor System.

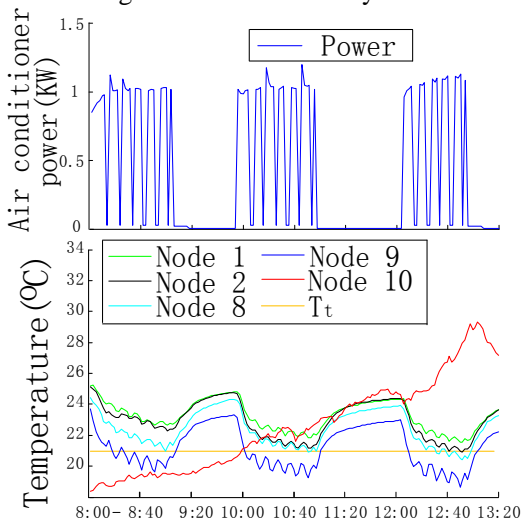

Figure 6: Example of Experiment Results

the equation $\mathcal{E}=f(\lambda, a, b, c, \ldots)$, see more details in [29].

We develop a wireless sensor network to collect these data for inverse calibration. The sensor network collect indoor temperature, outdoor temperature and electricity usage. Similar ideas have been proposed in [22] and our previous work [13][38]. We release our code and EnergyPlus bag in [29]. Due to page limitation, we omit the details of formal derivation and explanation of equation groups $\mathcal{E}=f(\lambda, a, b, c, \ldots)$.

\section{B. Wireless Sensor Network Design}

The objective of our wireless sensor network is to collect the electricity usage to air-conditioning the room, and indoor, outdoor temperatures.

We develop a two tiered WSNs as shown in Fig. 5. The end tier is a set of TelosB-based temperature sensors. They record temperatures and send such readings to the top tier, the Imote2-based electricity-meters. The Imote2 is extended with an electricity meter. As such, it can record and send electricity usage in real time. We also developed a long-range data communication module (LR-Module, in connection through $3 \mathrm{G}$ or $\mathrm{WiFi}$ ) and connect it to the Imote2. As such, the data can be transmitted to a remote base station. This is because we cannot place the base station (e.g. laptop computer), unattended, in the rooms of experiments and one cannot afford to always have people in the rooms of experiments. ${ }^{2}$

We implement our sensor system in TinyOS, and use Collection Tree Protocol (CTP) for data routing among sensor

\footnotetext{
${ }^{2}$ The Imote 2 sensors and the TelosB sensors are less conspicuous; they can be placed in boxes and hung on the walls.
} 


\begin{tabular}{c|cc}
\hline Period & $\begin{array}{c}\text { Measurement } \\
(\mathrm{kWh})\end{array}$ & $\begin{array}{c}\text { WSN+EnergyPlus } \\
(\mathrm{kWh})\end{array}$ \\
\hline 8:00-9:00 & 560 & 505 \\
10:00-11:00 & 690 & 755 \\
12:00-13:00 & 780 & 845 \\
17:00-18:00 & 530 & 480 \\
22:00 - 23:00 & 510 & 560 \\
\hline
\end{tabular}

Table I: Measured vs. Simulated Energy Consumption

nodes. The Imote 2 sends the temperature data collected from the end tier, and its electricity readings to our remote base station. The Imote2 has high load to relay data, but it has direct power supply and TelosB sensors use batteries.

\section{Validation}

We conduct real experiments to validate our inverse calibration using wireless sensor network. The configuration of the room of our experiments and the sensor network deployment are shown in [29]. We deployed nine indoor sensors, one outdoor sensor to collect temperature and an electricity-meter connected to the air-conditioner (AC). In our experiments, we periodically turned on and off the AC. Fig. 6 shows part of our experiment data: the upper figure shows the electricity usage recorded and the lower figure shows the temperatures recorded. We compute $\bar{\lambda}$ by the average of the $\lambda$ s of a set of electricity and temperature data. After we have $\bar{\lambda}$ and other parameters, we can put them into EnergyPlus to compute energy requirements of a room.

To validate the accuracy of our method, we use $\bar{\lambda}$ to simulate the energy consumption in five periods when the $\mathrm{AC}$ is in operation. We show the results in Table. I. There are two columns. The 1 st column shows the real measured data, and the 2nd column shows the data by $\bar{\lambda}$ assisted EnergyPlus computation. The errors are around 9\%. Note that the purpose of our physical modeling is not to achieve ultimate accuracy and make contribution to thermodynamic theory, but inputs that are reasonable enough for our computing algorithms.

\section{AlgORITHM}

Our philosophy in developing the heuristic for MBEC is as follows. We need to develop two schedules, 1) the meeting schedule and the room assignment schedule and 2) the electricity demand schedule. Accordingly, we develop two algorithms: 1) given the electricity demand schedule fixed, find the best meeting schedule and room assignment schedule; we call it algorithm best-Assignment(), and 2) given the meeting schedule and room assignment schedule fixed, find the best electricity demand schedule; we call it algorithm best-Demand(). We solve the overall MBEC by a Lagrangian relaxation structure using best-Demand() and best-Assignment() as sub-routines.

Given the meeting and room schedule fixed, finding the best electricity purchasing schedule (i.e., best-Demand()) can be optimally solved as it can be transformed into linear

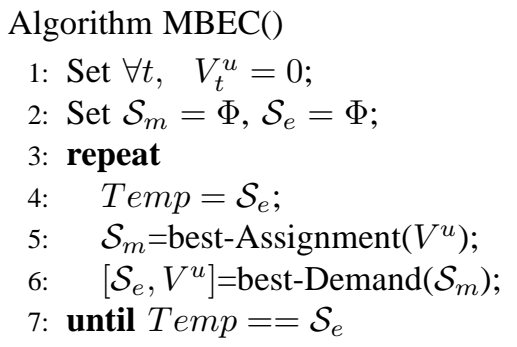

Figure 7: Algorithm MBEC.

programming. Given the electricity purchasing schedule fixed, finding meeting and room assignment schedule (bestAssignment()) is NP-complete.

In what follows, we will mainly discuss how we develop best-Assignment(); and how best-Demand() and bestAssignment() interact to solve MBEC.

Note that if there is no thermal storage, a meeting schedule and the room assignment schedule computed by best-Assignment() can determine the electricity purchasing schedule. We define usable thermal storage as the thermal storage volume that can be used at a time. Intuitively, usable thermal storage is the flexible storage volume at a time. With different usable thermal storage volume, a fixed meeting schedule and the room assignment schedule can reflect different electricity purchasing schedule. This usable thermal storage provides a linkage between best-Assignment() and best-Demand(). The inputs of best-Assignment() are usable thermal storage and meeting requirements. Its output is a meeting and room assignment schedule. The input of bestDemand() is a meeting and room assignment schedule and its output is electricity purchasing schedule and the possible usable thermal storage.

Algorithm MBEC() is shown in Fig. 7. Algorithm MBEC() first calls best-Assignment() where the input of usable thermal storage $V^{u}$ is 0 . It determines a meeting and room assignment schedule $\mathcal{S}_{m}$. $\mathcal{S}_{m}$ is given to best-Demand(). best-Demand() will compute the electricity purchase schedule $\mathcal{S}_{e}$ according to $\mathcal{S}_{m}$ and adjust the usable thermal storage $V^{u}$. Such $V^{u}$ is returned to bestAssignment(). The ending condition for $\operatorname{MBEC}()$ is if there is no change in the schedules.

Algorithm best-Assignment() is greedy-oriented. We first group the rooms according to its capacity, and sort room groups in descending order according to capacity. Second, we classify the meetings into different meeting groups according to room groups. For example, if we have room groups of capacity 20 and 40, we classify meetings of 25 people into meeting group of 40. As such, we have corresponding group pairs, i.e., the room group and meeting group. In each group pair, we calculate the cost and assign meetings to the rooms greedily, i.e., from the smallest cost one to the largest one. 


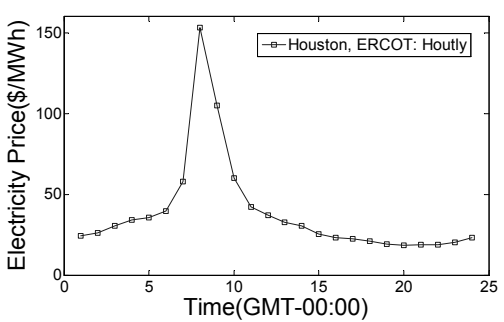

(a)

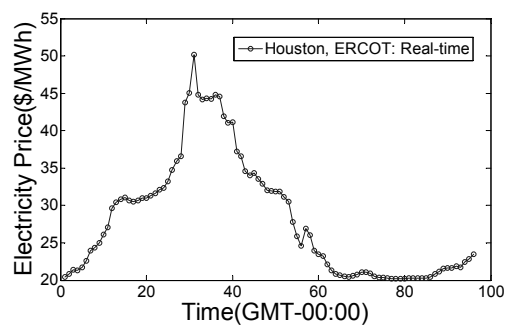

(b)

Figure 8: Comparison price differ for day-ahead market and real-time market in Houston on Aug 30, 2012. (a) Day-ahead market (b) Real-time market.

\section{SimULATION}

\section{A. Simulation Setup}

We evaluate our algorithms using real electricity price data, synthetic room configurations we generate based on our validation in Section VI and synthetic meeting requirements. We discuss each of these in details.

We first comment on the electricity price. We obtained the electricity price data of Houston from ERCOT (Electric Reliability Council of Texas). It has a day-ahead market and a real-time market. The day ahead market is the predicted price from ERCOT for the next day. This is not the true price as the true price is real-time that subjects to the real demands. Nevertheless, the trend of the day-ahead market and real-time market matches well. We show the day-ahead market and real-time price at Houston on August 30th, 2012 in Fig. 8 (a) and (b). We can see that the peak of real-time price is usually smaller than the day-ahead prediction; this shows that the intimidating day-ahead high predicted price can reduce demand to certain level. We also see that the real-time price and day-ahead price share the same trend. This implies that if we develop our schedules using dayahead price (in other words, these are offline schedules), we will obtain reasonable good result even if we do not have real-time price. In our simulation, our evaluation is based on real-time price and we will compare with day-ahead price. The dynamic price adjustment interval is 15 minutes. We also show the daily electricity price from real-time market in August, 2012 in Fig. 9. These data can be found from [35] and similar evaluation setup has been used in [9][12].

The room configurations are summarized in Table II. The total number of rooms is 110 . (S) and $(\mathrm{N})$ represent the orientation of room, i.e., south and north. In general, rooms towards south have higher energy consumption for air-conditioning. We assume the materials of walls, floor and ceiling in the rooms are same to the materials of the hotel rooms in our validation (Section VI). As a consequence, we can calculate the $\lambda$ based on room size, position and orientation. We then use EnergyPlus to compute the energy consumption of each room each hour. The $P$ in the Table shows the median and the variance of the results.

We set the target temperature $T_{t}=23.5^{\circ} \mathrm{C}\left(74.3^{\circ} \mathrm{F}\right)$ for all meetings, the standard temperature recommended for

\begin{tabular}{c|cccc}
\hline $\begin{array}{c}\text { Cap } \\
(\mathrm{S} / \mathrm{N})\end{array}$ & Num & $\begin{array}{c}\text { Size } \\
(L \times W \times H)\end{array}$ & $\begin{array}{c}\lambda \\
(\mathrm{J} / \mathrm{s} \cdot K)\end{array}$ & $\begin{array}{c}P \pm 20 \% \\
(\mathrm{~kW})\end{array}$ \\
\hline $20(\mathrm{~S})$ & 10 & $4 \times 5 \times 3$ & 49.8 & 1.5 \\
$20(\mathrm{~N})$ & 10 & $4 \times 5 \times 3$ & 40.2 & 1.2 \\
$40(\mathrm{~S})$ & 20 & $8 \times 5 \times 3$ & 83.7 & 2.4 \\
$40(\mathrm{~N})$ & 20 & $8 \times 5 \times 3$ & 63.2 & 1.8 \\
$60(\mathrm{~S})$ & 20 & $6 \times 10 \times 3$ & 114.5 & 4.7 \\
$60(\mathrm{~N})$ & 20 & $6 \times 10 \times 3$ & 82.5 & 3.3 \\
$80(\mathrm{~S})$ & 5 & $8 \times 10 \times 3$ & 142.0 & 6.2 \\
$80(\mathrm{~N})$ & 5 & $8 \times 10 \times 3$ & 118.5 & 4.9 \\
\hline
\end{tabular}

Table II: Room configuration

Grade A buildings in Hong Kong. We set the meetings from [8:00, 22:00] in each day. The length of the meetings are randomly selected from two groups, $\mathcal{O}_{1}=[1,1.5,2,2.5,3]$, $\mathcal{O}_{2}=[1,2,3]$. For example, for $\mathcal{O}_{1}$, the meeting lengths are randomly chosen from $1,1.5,2,2.5$ or 3 hours. As a reference, if the meetings are all 2 hours, the total number of meetings the building can hold in one day is 770 . The meeting capacity requirement is set randomly but proportional to the room capacity. Similar evaluation setup can be found in [13].

We compare our algorithm MBEC with 1) room scheduling algorithm that just satisfies the meeting time and room capacity requirements (denoted as just-fit). We have consulted the class scheduling of our university and there is no special algorithm designed with considerations on energy or electricity issues. Therefore, we believe just-fit can be considered as a standard benchmark; and 2) bestAssignment() only.

\section{B. Simulation Result}

In the simulation, we first consider the impact of meetings, prices and thermal storage on electricity costs. We then evaluate our algorithms used for $\mathrm{f}-\mathrm{MBEC}()$ and compare to $\operatorname{MBEC}()$. At the end of section, we further evaluate our algorithms using day-ahead price. As a comparison benchmark, the electricity cost if all rooms are fully assigned (i.e., from 8:00 - 22:00) is $\$ 173.3$.

The default values for thermal storage is set to $500 \mathrm{kWh}$; this can approximately support all rooms for 1.5 hours. We use $\mathcal{O}_{1}$ as our default meeting length option group. The cost saving is the difference between $\operatorname{MBEC}()$ and just-fit 


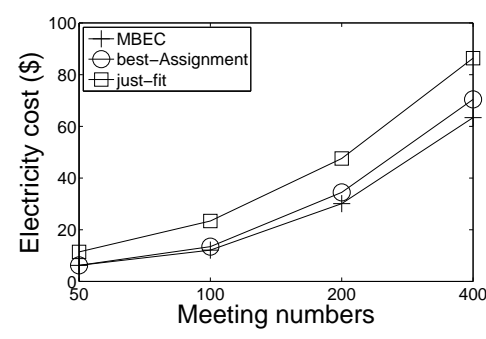

(a)

Figure 10: Total electricity cost of rooms as against to the number of meetings. (a)meeting length: option $\mathcal{O}_{1}$ (b)meeting length: option $\mathcal{O}_{2}$.

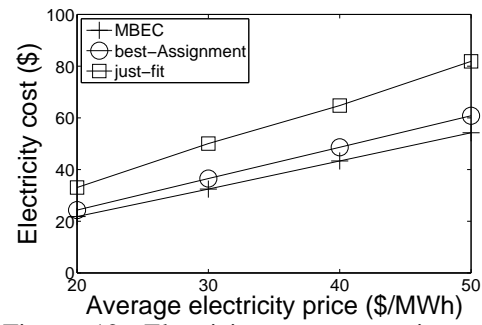

Figure 12: Electricity cost as against to average electricity price.

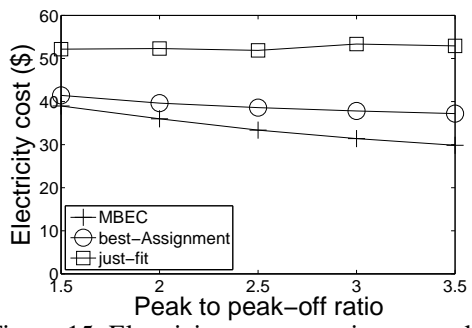

Figure 15: Electricity cost as against to peak to peak-off ratio.

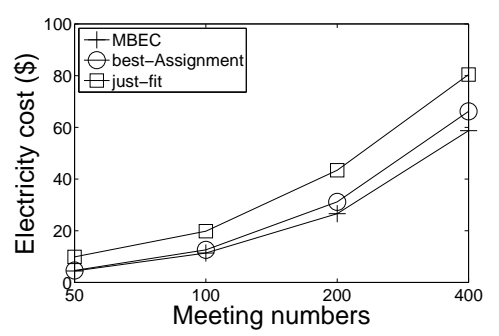

(b)

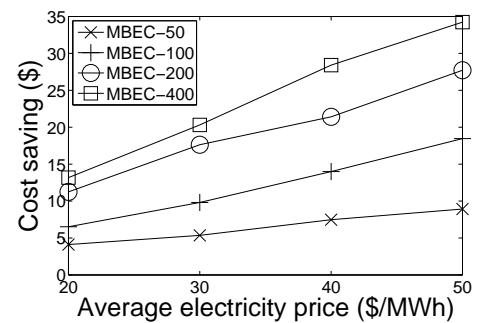

Figure 13: Cost saving as against to average electricity price.

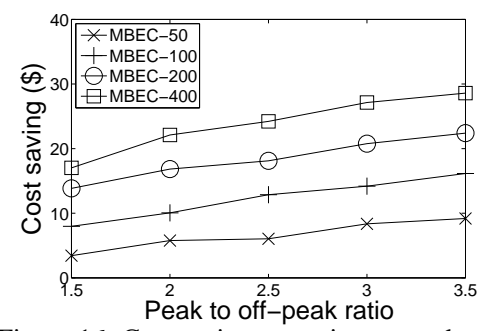

Figure 16: Cost saving as against to peak to peak-off ratio.

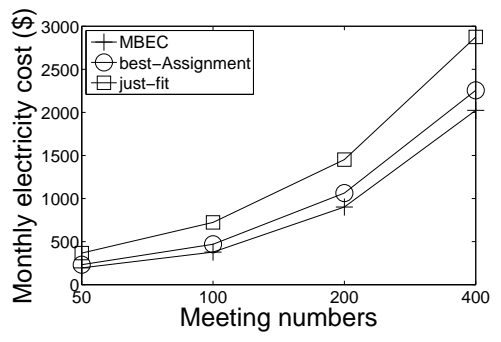

Figure 11: Monthly electricity cost as against to the number of meetings.

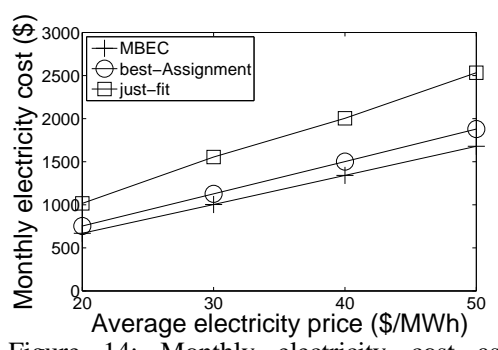

Figure 14: Monthly electricity cost as against to average electricity price.

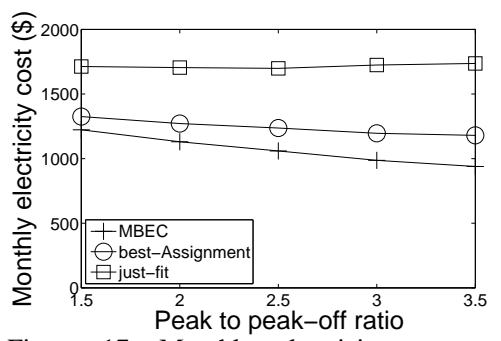

Figure 17: Monthly electricity cost as against to peak to peak-off ratio. scheduling.

1) Impact of Meeting Configuration: Fig. 10 (a) show the electricity cost as a function of meeting numbers. For all three algorithms, we can see that if there are more meetings (i.e., more human activities), there needs more costs. We can also see that both our algorithms best-Assignment() and MBEC() save costs as compared to the just-fit. This is not surprising as the just-fit schedule only satisfies the meeting capacity requirement. Specifically, we see that if there are 200 meetings, the electricity cost needed by just-fit, bestAssignment() and MBEC() is \$47.5, \$34.5 and \$30.0. Our algorithm MBEC() has a saving of $36.8 \%$. Note that such saving is achieved only by more careful scheduling. Fig. 10 (b) shows very similar results if the meeting length option is in $\mathcal{O}_{2}$.

Fig. 11 extends the results to the full month of Aug. 2012. As the same as the daily electricity cost, both our algorithms best-Assignment() and $\operatorname{MBEC}()$ save costs as compared to the just-fit schedule. If there are 200 meetings, the total monthly electricity cost needed by just-fit, bestAssignment() and MBEC() is \$1441.7, \$1064.0 and \$894.5 and MBEC() saves $40.0 \%$.
2) Impact of Dynamic Price: There are two important parameters for the dynamic pricing: 1) average price and 2) peak to off-peak ratio. We study both these situations. First, we adjust the average price of our electricity price data while keeping peak to off-peak ratios constant. In our baseline situation, the average price is $32.4 \$$. We adjust this to a range of $[20,50]$. Second, we adjust the peak to offpeak ratio while keeping the average price constant. In our baseline situation, the peak to off-peak ratio is 2.2 . We adjust this to $[1.5,3.5]$.

Fig. 12 shows the electricity cost as against to the average price when meeting number is 200 . For all three algorithms, we can see that if there are higher average electricity price, there are higher costs. This figure shows that the total electricity cost increases when the average price increases; yet the just-fit scheduling increases faster than our algorithms.

In Fig. 13 we show the cost savings as against to the average price. Clearly, MBEC() outperforms ad-hoc scheduling and we want to evaluate the gap under different situations. We see that the more meetings we have, the larger the gap is. We also see that when average electricity price increases, we have more savings. Fig. 14 show the monthly electricity 


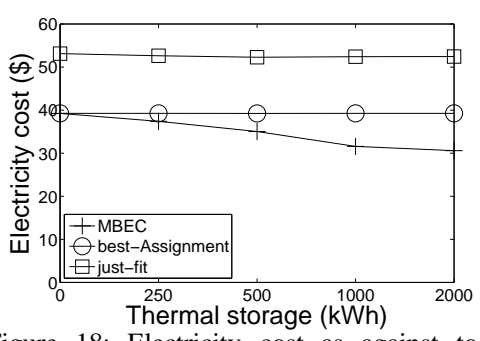

Figure 18: Electricity cost as against to thermal storage.

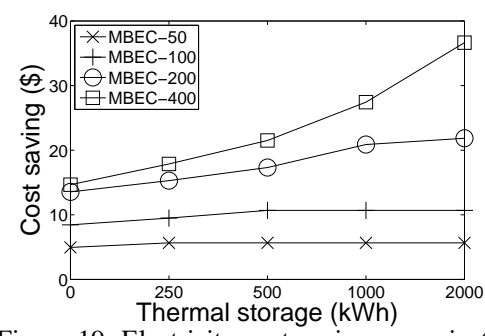

Figure 19: Electricity cost saving as against to thermal storage.

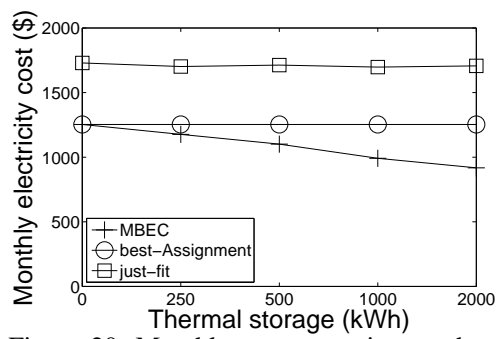

Figure 20: Monthly cost as against to thermal storage.

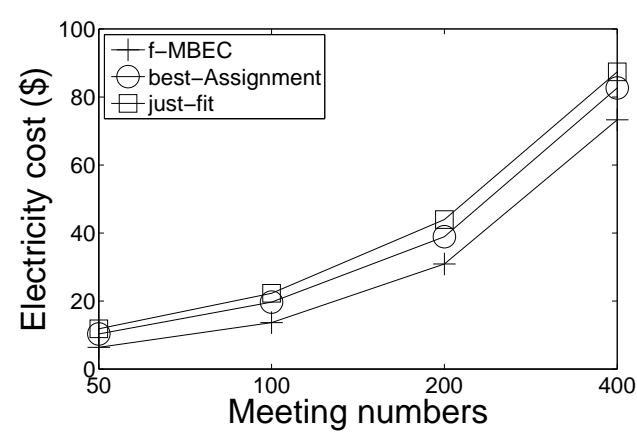

Figure 21: Electricity cost as against to the number of meetings when meetings have fixed start and end time.

cost as against to the average price when meeting number is 200 . We see the same results as the daily electricity cost.

Fig. 15 shows the electricity cost as against to the peak to off-peak price when the number of meetings is 200 . We can see that if the peak to off-peak ratio increases, the total electricity cost of just-fit scheduling stays the same and our algorithms decrease. Clearly, just-fit scheduling is ignorant to the cost and our algorithms can take more advantages of the cost differences. When the peak to peak-off ratio is 3, MBEC() can outperform just-fit for as much as $39.8 \%$. In Fig. 16 we show the cost savings as against to the peak to off-peak ratio and we see similar results.

Fig. 17 shows the monthly electricity cost when the number of meetings is 200 . We compare all three algorithms. Again, the same as the daily electricity cost, both best-Assignment() and MBEC() substantially save costs as compared to the just-fit scheduling.

3) Impact of Thermal Storage: Fig. 18 shows the electricity cost as against to thermal storage capacity when the number of meetings is 200 . We can see that if the thermal storage capacity increases, the electricity costs of just-fit scheduling and best-Assignment() do not change, while bestAssignment() is better. The electricity costs of MBEC() keep decreasing. This is not surprising as just-fit scheduling and best-Assignment() do not use the thermal storage for cost saving. When the thermal storage is $1000 \mathrm{kWh}$ (approximately support all rooms for 3 hours), it can introduce a saving of $22.3 \%$ as compared to best-Assignment().

Fig. 19 shows the saving cost when we use different thermal capacity and Fig. 20 show the monthly electricity cost as against to thermal storage capacity. All these show that having an appropriate thermal storage capacity is very cost-effective.

4) Meetings with Fixed Start and End Time: In many scenarios, meetings have fixed start and end time. We specially evaluate this in this subsection. Our algorithm MBEC() can naturally adapt to this. We call it f-MBEC() in what follows to make the context clearer. Note that if the start and end times are fixed, these times become constraints (inputs for the algorithm) rather than to be computed. In our simulation, we randomly generate the start and end times for the meetings, The length of the meetings are randomly selected from the meeting length group $\mathcal{O}_{1}$.

Fig. 21 show the results. Similarly, we see that if there are more meetings, there needs more costs and both bestAssignment() and f-MBEC() save costs as compared to the just-fit schedule. Compared to flexible start and end time, the saving becomes smaller, yet f-MBEC() still has a saving of around $25 \%$.

\section{CONCLUSION}

In this paper, we studied minimizing electricity bills of buildings in a dynamic power market. We presented a holistic planning by developing electricity purchasing schedule from the power market on one end and meeting schedules and room assignment schedules for the building to support human activities on the other end. The thermal storage plays a key role in cost reduction and linkage between the supply and demand.

Our problem is cross-disciplinary in nature and we developed both computing algorithms and physical modeling, which is assisted by our wireless sensing systems. We showed real experiments for validation. We observed that minimizing electricity bills does not coincide with minimizing energy consumption. We studied the cause and their relationship. Unfortunately, we believe that the incentive of the building operators is to reduce costs. We would like to conduct a in-depth study on an appropriate balance of them in the future.

\section{ACKNOWLEDGMENT}

Dan Wang's work is supported in part by National Natural Science Foundation of China (No. 61272464), RGC/GRF PolyU 5264/13E, HK PolyU G-YM06, A-PK95. 


\section{REFERENCES}

[1] H. K. Nguyen, J. B. Song, and Z. Han, "Demand side management to reduce peak-to-average ratio using game theory in smart grid," in Proc. INFOCOM WKSHPS, 2012.

[2] X. Fang, S. Misra, G. Xue, and D. Yang, "Smart gridłthe new and improved power grid: A survey," IEEE Communications Surveys and Tutorials, pp. 944-980, 2012.

[3] W. Wang, Y. Xu, and M. Khanna, "A survey on the communication architectures in smart grid," Computer Networks, pp. 3604-3629, 2011.

[4] "Energy in the united states," 2010, http://en.wikipedia.org/wiki/Energy_in_the_United_States.

[5] "Hong kong energy end-use data," Electrical and Mechanical Service Department, Hong Kong, Tech. Rep., 2010, http://www.emsd.gov.hk/emsd/e_download/pee/HKEEUD2010.pdf.

[6] M. Behl, T. Nghiem, and R. Mangharam, "Green scheduling for energy-efficient operation of multiple chiller plants." in RTSS, 2012.

[7] D. Yang, L. Xu, S. Gong, H. Li, G. Peterson, and D. Zhang, "Joint electrical load modeling and forecasting based on sparse bayesian learning for the smart grid," in Proc. CISS, 2011.

[8] A. Qureshi, R. Weber, H. Balakrishnan, J. Guttag, and B. Maggs, "Cutting the Electric Bill for Internet-Scale Systems," in Proc. ACM SIGCOMM, 2009.

[9] J. Li, Z. Li, K. Ren, and X. Liu, "Towards optimal electric demand management for internet data centers," IEEE Trans. Smart Grid, vol. 3, no. 1, pp. 183-192, 2012.

[10] J. Yao, X. Liu, W. He, and A. Rahman, "Dynamic control of electricity cost with power demand smoothing and peak shaving for distributed internet data centers," in Proc. ICDCS, 2012.

[11] B. Daryanian, R. Bohn, and R. Tabors, "An experiment in real time pricing for control of electric thermal storage systems," IEEE Trans. Power Systems, vol. 6, no. 4, pp. 1356-1365, 1991.

[12] A. Mishra, D. Irwin, P. Shenoy, J. Kurose, and T. Zhu, "Smartcharge: cutting the electricity bill in smart homes with energy storage," in Proc. e-Energy, 2012.

[13] D. Pan, Y. Yuan, D. Wang, X. Xu, Y. Peng, X. Peng, and P. Wan, "Thermal inertia: Towards an energy conservation room management system," in Proc. INFOCOM, 2012.

[14] A. Majumdar, D. H. Albonesi, and P. Bose, "Energy-aware meeting scheduling algorithms for smart buildings," in Proc. BuildSys, 2012.

[15] Y. Li, R. Kaewpuang, P. Wang, D. Niyato, and Z. Han, "An energy efficient solution: Integrating plug-in hybrid electric vehicle in smart grid with renewable energy," in Proc. INFOCOM WKSHPS, 2012.

[16] S. Pacala and R. Socolow, "Stabilization wedges: solving the climate problem for the next 50 years with current technologies," science, vol. 305, no. 5686, pp. 968-972, 2004.

[17] O. Gnawali, R. Fonseca, K. Jamieson, D. Moss, and P. Levis, "Collection tree protocol," in Proc. SenSys, 2009.

[18] X. Jiang, M. Van Ly, J. Taneja, P. Dutta, and D. Culler, "Experiences with a high-fidelity wireless building energy auditing network," in Proc. SenSys, 2009.

[19] S. Dawson-Haggerty, X. Jiang, G. Tolle, J. Ortiz, and D. Culler, "smap: a simple measurement and actuation profile for physical information," in Proc. SenSys, 2010.
[20] L. Schor, P. Sommer, and R. Wattenhofer, "Towards a zeroconfiguration wireless sensor network architecture for smart buildings," in Proc. BuildSys, 2009.

[21] Q. Luo, A.-Y. Lam, D. Wang, D.-T. Chan, Y. Peng, and $\mathrm{X}$. Peng, "Demo abstract: Towards a wireless building management system with minimum change to the building protocols," in Proc. ICCPS, 2012.

[22] A. Aswani, N. Master, J. Taneja, D. Culler, and C. Tomlin, "Reducing transient and steady state electricity consumption in hvac using learning-based model-predictive control," Proc. the IEEE, vol. 100, no. 1, pp. $240-253,2012$.

[23] K. Deng, P. Barooah, P. Mehta, and S. Meyn, "Building thermal model reduction via aggregation of states," in American Control Conference, 2010.

[24] J. Chen, R. Tan, Y. Wang, G. Xing, X. Wang, X. Wang, B. Punch, and D. Colbry, "A high-fidelity temperature distribution forecasting system for data centers," in RTSS, 2012.

[25] Z. Li, P.-C. Huang, A. K. Mok, T. Nghiem, M. Behl, G. J. Pappas, and R. Mangharam, "On the feasibility of linear discrete-time systems of the green scheduling problem," in RTSS, 2011.

[26] T. Hnat, V. Srinivasan, T. Sookoor, R. Dawson, J. Stankovic, and K. Whitehouse, "The hitchhiker's guide to successful residential sensing deployments," in Proc. SenSys, 2011.

[27] Z. Yang, N. Li, B. Becerik-Gerber, and M. Orosz, "A nonintrusive occupancy monitoring system for demand driven hvac operations," in Proc. CRC, 2012.

[28] V. Erickson and A. Cerpa, "Thermovote: Participatory sensing for efficient building hvac conditioning," in Proc. BuildSys, 2012.

[29] D. Pan, D. Wang, J. Cao, Y. Peng, and X. Peng, "Minimizing building electricity costs in a dynamic power market: Algorithms and impact on energy conservation," Tech. Rep., 2012, available at http://www4.comp.polyu.edu.hk/ csdwang/Projects/wBACnet.htm.

[30] "Getting started with energyplus," U.S. Department of Energy, Tech. Rep., 2010.

[31] D. Chan, J. Burnett, R. de Dear, and S. Ng, "Large-scale survey of thermal comfort in office premises in hong kong," ASHRAE Transactions, 1998.

[32] F. Spieksma, "On the approximability of an interval scheduling problem," J. Scheduling, vol. 2, no. 5, pp. 215-227, 1999.

[33] Q. Huang and E. Lloyd, "Cost constrained fixed job scheduling," Theoretical Computer Science, pp. 111-124, 2003.

[34] A. Mohsenian-Rad, V. Wong, J. Jatskevich, R. Schober, and A. Leon-Garcia, "Autonomous demand-side management based on game-theoretic energy consumption scheduling for the future smart grid," IEEE Trans. Smart Grid, vol. 1, no. 3, pp. 320-331, 2010.

[35] "Gdf suez energy resources[online]," available: http://www.gdfsuezenergysources.com.

[36] W. Bernal, M. Behl, T. X. Nghiem, and R. Mangharam, "Mle+: a tool for integrated design and deployment of energy efficient building controls," in Proc. BuildSys, 2012.

[37] P. Ihm, M. Krarti, and G. P. Henze, "Development of a thermal energy storage model for energyplus," Energy and Buildings, vol. 36, pp. 807-814, 2004.

[38] Y. Yuan, D. Pan, D. Wang, X. Xu, Y. Peng, X. Peng, and P. Wan, "A study towards applying thermal inertia for energy conservation in rooms," to appear in ACM Trans. on Sensor Networks, 2013. 